Pacific Journal of Mathematics

ON THE EIGENVALUES OF A SECOND ORDER ELLIPTIC 


\section{ON THE EIGENVALUES OF A SECOND ORDER ELLIPTIC OPERATOR IN AN UNBOUNDED DOMAIN}

\section{Denton HewgILL}

Let $E$ be an open set in $R^{n}$ which satisfies the "narrowness at infinity" condition:

$$
\text { meas }\left(E \cap\left\{x \in R^{n}: a \leqq|x|<a+1\right\}\right) \leqq \operatorname{const}(a+1)^{-\beta},
$$

for all $a>0$ and some $\beta>0$. It is known that a uniformly strongly elliptic self-adjoint partial differential operator, on such a set $E$, has a discrete spectrum of eigenvalues $\left\{\lambda_{j}\right\}$. This paper is concerned with the growth rate of the function

$$
N(\lambda)=\sum_{\lambda \leqq \lambda} 1 \text {. }
$$

The main result of the paper is to give an upper bound for $N(\lambda)$. This upper bound will be a function of the $\beta$ from the "narrowness" condition.

An unbounded open set $E$ in Euclidean $n$-space $R^{n}$ is said to be quasi-bounded if the points $x \in E$ with $|x|$ large are near the boundary $\partial E$ :

$$
\lim _{x \rightarrow \infty, x \in E} \operatorname{dist}(x, \partial E)=0 .
$$

Let $T$ be the $L_{2}(E)$-realization of the uniformly strongly elliptic second order partial differential operator $a(x, D)$ with zero Dirichlet boundary conditions:

$$
\begin{gathered}
a(x, D)=-\sum_{|\alpha| \leq 2} a_{\alpha}(x) D^{\alpha}, \quad D^{\alpha}= \\
\left(\partial / \partial x_{1}\right)^{\alpha_{1}} \cdots\left(\partial / \partial x_{n}\right)^{\alpha_{n}}, \\
|\alpha|=\left|\alpha_{1}\right|+\cdots+\left|\alpha_{n}\right|, \\
a_{0}(x, \xi) \geqq \mathrm{const}|\xi|^{2 m}, \quad x \in R^{n}, \quad \xi \in R^{n}
\end{gathered}
$$

where $a_{0}(x, \xi)$ is the principle part of $a(x, \xi)$; the coefficients $a_{\alpha}(x)$ are infinitely differentiable bounded real functions in $R^{n} ; a(x, D)$ is formally self-adjoint;

$$
\begin{gathered}
\mathscr{D}(T)=H_{0}^{1}(E) \cap\left\{f \in L_{2}(E): a(x, D) f \in L_{2}(E)\right\} \\
T f=a(x, D) f, \quad f \in \mathscr{D}(T),
\end{gathered}
$$

where $H_{0}^{1}(E)$ is the standard Sobolev space. If $E$ is quasi-bounded and satisfies some additional smoothness conditions, that it is known, Clark [4] and Adams [1], that $T$ has a compact resolvent, and thus a discrete spectrum, consisting of eigenvalues $\lambda_{j}$ satisfying 


$$
0<\lambda_{1} \leqq \lambda_{2} \leqq \cdots \lambda_{j} \longrightarrow \infty \text { as } j \longrightarrow \infty \text {. }
$$

Define the "trace function" by

$$
N(\lambda)=\sum_{\lambda \leq \lambda} 1
$$

For $a(x, D)$ equal to the Laplacian, the asymptotic nature of $N(\lambda)$ was discussed by: Weyl for $E$ a bounded set; Clark [5] for $E$ an unbounded set with finite volume; Clark and Hewgill [6] for $E$ with an infinite volume.

In this paper we consider domains which satisfy a $\beta$-condition:

$$
\text { meas }(E \cap\{x: a \leqq x<a+1\}) \leqq C(a+1)^{-\beta}, \quad a \geqq 0,
$$

where $\beta$ and $C$ are positive constants. The main result we shall prove is theorem. If $4 k>\beta^{-1}$ when $\operatorname{dim} E=2$, and $2 k>\beta^{-1}$ when $\operatorname{dim} E=3$, then

$$
N(\lambda) \leqq \text { const } \lambda^{4 k}, \quad \lambda>0 .
$$

This theorem will generalize, to an elliptic operator, some of the results stated in Hewgill [8] and Rozenbljum [11].

The method we shall use is to construct a fundamental singularity of a special type which will estimate the Green's function for the problem. Then, we prove that some iterate of the Green's function is a Hilbert-Schmidt kernel, from which our upper bound on $N(\lambda)$ will follow.

1. The fundamental singularity and the Green's function. We take as a starting point Gårding's paper [7], which constructs a fundamental singularity for $T$ in an unbounded domain. We remark here that Garding's construction of the Green's function and his estimates for it will not work in an unbounded domain. Gårding's results to be used are summarized in the following theorem:

THEOREM 1.1. Let the coefficients of the uniformly strongly elliptic operator $a(x, D)$ be infinitely differentiable functions in $R^{n}$. Then, there exists a function $\Gamma(\tau, z, x)$, the fundamental singularity for $a(x, D)$, such that the following conditions hold:

$$
\Gamma(\tau, z, x)=O(1) \tau^{n} e_{0}(\tau(x-z))\left(1+|\tau(x-z)|^{N}\right)^{-1}, \quad x, z \in R^{n},
$$

where $e_{0}(y)=|y|^{2-n-\varepsilon}$ for $n \geqq 2$;

$$
\begin{gathered}
\left(a(x, D)+\tau^{2}\right) \Gamma(\tau, z, x)=0, \quad x \neq z ; \\
\tau^{-2}\left(a(x, D)+\tau^{2}\right) \Gamma f(x)=f(x)
\end{gathered}
$$

where 


$$
\Gamma f(x)=\int \Gamma(\tau, z, x) f(z) d z, \quad f \in C_{0}^{\infty}\left(R^{n}\right) .
$$

In these formulas $N>0$ and $1>\varepsilon>0$ are arbitrary, and the estimate $O(1)$, for large $\tau$, is uniform in $R^{n} \times R^{n}$.

In the case where $a(x, D)$ is the Laplace operator, Brownell [3] has shown that the fundamental singularity has exponential decay in $R^{n}$.

Next, we need a Green's function $G_{R}(\tau, z, x)$ for the bounded cut off domain $E_{R}=\{x \in E:|x|<R\}$. Krzyżański [9] shows:

THEOREM 1.2. For $\tau$ sufficiently large and positive, there exists a Green's function $G_{R}(\tau, z, x)$, which is continuous in $\operatorname{cl} E_{R} \times \operatorname{cl} E_{R}$ when $x \neq z$

$$
G_{R} f(x)=\int_{E_{R}} G_{R}(\tau, z, x) f(z) d z, \quad f \in C_{0}^{\infty}\left(E_{R}\right),
$$

has continuous second derivatives in $E_{R}$;

$$
\left(a(x, D)+\tau^{2}\right) G_{R} f(x)=f(x), \quad f \in C_{0}^{\infty}\left(E_{R}\right) ;
$$

and $G_{R} f(x)=0$ for $x$ in the boundary of $E_{R}$.

Lemma 1.3. If $\operatorname{dim} E$ is 2 or 3 , then the function $\Gamma f(x)$, defined for $f \in C_{0}^{\infty}\left(R^{n}\right)$ by Theorem 1.1, tends to zero as $|x| \rightarrow+\infty$.

Proof. By Theorem 1.1,

$$
\Gamma(\tau, z, x)=O(1) \tau^{n}|\tau(x-z)|^{2-n-\varepsilon}\left(1+|\tau(x-z)|^{2 N}\right)^{-1},
$$

for arbitrary $N$ and $0<\varepsilon<1$. If $f \in C_{0}^{\infty}\left(R^{n}\right)$, then

$$
\begin{gathered}
|\Gamma f(x)| \leqq\left\{\sup _{z \in \operatorname{supp} f} 2\left(1+|\tau(x-z)|^{N}\right)^{-1}\right\} \int_{R^{n}} O(1) \tau^{n}|\tau(x-z)|^{2-n-\varepsilon} \\
\left(1+\left|\tau(x-z)^{N}\right|\right)^{-1}|f(z)| d z,
\end{gathered}
$$

by use of the elementary inequality $(a+b)^{2} \leqq 2 a^{2}+2 b^{2}$.

Since $|f(z)|$ is bounded, the above integral is independent of $x$ by translation, and is convergent if $N$ is chosen larger than $n+1$ with $\operatorname{dim} E$ equal to 2 or 3 . Since $f$ has bounded support, the function $\Gamma f(x) \rightarrow 0$ as $|x| \rightarrow \infty$.

Lemma 1.4. The fundamental solution $\Gamma(\tau, z, x)$ is nonnegative in $R^{n} \times R^{n}$.

Proof. Assume $\Gamma\left(\tau, z_{0}, x_{0}\right)$ is negative for some $x_{0}$ and $z_{0}$ with $x_{0} \neq z_{0}$. Then, there is a function $f$, which is nonnegative and has 
small support, such that $\Gamma f\left(x_{0}\right)<0$. Let $\varepsilon=\Gamma f\left(x_{0}\right) / 2$. By Lemma 1.3 , we have a sphere $K$ such that $|\Gamma f(x)| \leqq \varepsilon$ in $R^{n}-K$. Also

$$
\left[-a(x, D)-\tau^{2}\right] \Gamma f(x)=-\tau^{2} f(x) \leqq 0,
$$

by Theorem 1.1. Thus the Hopf theorem implies that $\Gamma f(x) \geqq-\varepsilon$ in all of $R^{n}$, which is a contradiction and so the lemma is proved.

LEMma 1.5. The Green's function for the cut off domain $E_{R}$, given in Theorem 1.2, satisfies the inequality

$$
0 \leqq G_{R}(\tau, z, x) \leqq \Gamma(\tau, z, x), \quad x, z \in E_{R}
$$

Proof. The proof follows directly from a version of the maximum theorem given in Krzyżański [9, p. 436], by considering the function

$$
V(\tau, z, x)=\Gamma(\tau, z, x)-G_{R}(\tau, z, x),
$$

and using the properties of $\Gamma$ and $G_{R}$ proved in Lemmas 1.1, 1.2, 1.3, and 1.4 .

The next task is to discuss the Green's function for the whole domain $E$ and show its relation to the fundamental singularity $\Gamma$.

THEOREM 1.6. The operator $T$, which is defined on the open domain $E$ satisfying the $\beta$-condition, is a closed linear operator; the spectrum $\sigma(T)$ is discrete and has no finite limit points; the resolvent operator $R_{\lambda}(T)=(\lambda I-T)^{-1}$ is completely continuous; there is a complete set of eigenfunctions $\left\{u_{j}\right\}$ such that $T u_{j}=\lambda_{j} u_{j}$.

Proof. Since the $\beta$-condition on $E$ implies that $E$ is quasi-bounded, this is a special case of Clark's result [4, Theorem 5] for a uniformly strongly elliptic operator.

THeOREM 1.7. For $n=2$ and 3 the resolvent $R_{\lambda}(T)$ has a $L_{2}(E)$ Carleman kernel $G(\lambda, z, x)$, called the Green's function for $T$ on the domain $E$, such that

$$
\begin{gathered}
(\lambda I-T)^{-1} f(x)=-G f(x)=-\int_{E} G(\lambda, z, x) f(z) d z, \quad f \in L_{2}(E) \\
(\lambda-a(x, D)) G(\lambda, z, x)=0, \text { for } x \neq z
\end{gathered}
$$

and $\psi(\cdot) G(\lambda, z, \cdot) \in \mathscr{D}(T)$ for $\psi$ an infinitely differentiable function which is zero in a neighborhood of $z$ and equal to 1 in a boundary strip.

The above theorem is proved in Maurin [10, p. 244]. 
LemMA 1.8. Let the set $A$ have a compact closure in $E$, and $f \in C_{0}^{\infty}(A)$. Then, $G f=G_{R} f$ if $R$ is sufficiently large, where $G$ is the Green's operator for $E$ and $G_{R}$ is the Green's operator for the cut off domain $E_{R}$.

Proof. From Theorems 1.2 and 1.7 we have, for $R$ sufficiently large,

$$
\left(a(x, D)+\tau^{2}\right) G_{R} f(x)=f(x), \quad G_{R} f \in H_{0}^{1}\left(E_{R}\right),
$$

and

$$
\left(a(x, D)+\tau^{2}\right) G f(x)=f(x), \quad \lambda=-\tau^{2}, \quad G f \in H_{0}^{1}(E) .
$$

Now we extend $G_{R} f$ by zero so that it is in $H_{0}^{1}(E)$ and subtract the above equations to get

$$
\left(a(x, D)+\tau^{2}\right)\left[G f(x)-G_{R} f(x)\right]=0,
$$

with $G f-G_{R} f \in H_{0}^{1}(E)$. Thus the function $G f-G_{R} f$ is eigenfunction corresponding to the eigenvalue $-\tau^{2}$, however, $-\tau^{2}$ is not in the spectrum of the self-adjoint operator $T$ therefore $G f=G_{R} f$.

We finish this section with the following lemma.

LEMmA 1.9. The Green's function $G(\lambda, z, x)$ is continuous for $x \neq z$ and satisfies the inequality

$$
G\left(-\tau^{2}, z, x\right) \leqq \Gamma(\tau, z, x), \quad x \neq z, \text { for } \tau \text { sufficiently large } .
$$

Proof. Since the Green's function $G(\lambda, z, x)$ satisfies the equation $(\lambda-a(x, D)) G(\lambda, z, x)=0$ if $x \neq z$, the continuity of $G$ follows from a local regularity theorem for elliptic equations (see Agmon [2, Theorem 6.3]).

Now assume that there are two different points $\xi, \eta$ in $E$ such that

$$
G\left(-\tau^{2}, \xi, \eta\right)>\Gamma(\tau, \xi, \eta)
$$

Since both $G$ and $\Gamma$ are continuous there are two disjoint neighborhoods $U$ and $V$ of $\xi$ and $\eta$ respectively such that the same inequality holds. Let $f$ be a positive $C_{0}^{\infty}(U)$ function. Then, $G f(x)$ and $\Gamma f(x)$ satisfy the inequality

$$
G f(x)>\Gamma f(x), \quad x \in V,
$$

but Lemma 1.8 shows $G f(x)=G_{R} f(x)$ if $R$ is sufficiently large, therefore

$$
G_{R} f(x)>\Gamma f(x), \quad x \in V, R \text { large }
$$


However, this contradicts Lemma 1.5, and hence our theorem is proved.

2. The Hilbert-Schmidt properties of the Green's function. In this section we make essential use of the $\beta$-condition to show that some iterate of the Green's function is a Hilbert-Schmidt kernel.

Lemma 2.1. If $\alpha \geqq 0, \gamma\langle\beta, \tau\rangle \tau_{0}$, and $N$ is sufficiently large

$$
\left(N>\max \left\{2 n, 2(\alpha+\beta)(\beta-\gamma)^{-1}\right\}\right) \text {, }
$$

then

$$
\int_{E}(|x|+1)^{-\alpha}\left(1+|\tau(x-y)|^{N}\right)^{-1} d x<K(|y|+1)^{-\alpha-\gamma},
$$

where $K=K\left(\alpha, \beta, \gamma, \tau_{0}, N\right)$ but is independent of $y$.

Proof. Set $B(y, \delta)$ equal to the ball of radius $\delta$ about $y$ in $R^{n}$, $B(y)=B(y, \delta) \cap E$, and $B^{\prime}(y)=E-B(y)$. Further set $\delta$ equal to $(|y|+1)$ raised to the power $2(\alpha+\beta) / N$.

Consider first the integral in (1) taken over the set $B^{\prime}(y)$. To this integral we apply the inequality $(a+b)^{2} \leqq 2 a^{2}+2 b^{2}$ to get

$$
\begin{aligned}
& \int_{B^{\prime}(y)}(|x|+1)^{-\alpha}\left(1+|\tau(x-y)|^{N}\right)^{-1} d x \\
& \leqq 2\left(1+|\tau \delta|^{N / 2}\right)^{-1} \int_{R^{n}}\left(1+|\tau(x-y)|^{N / 2}\right)^{-1} d x .
\end{aligned}
$$

The integral on the right hand side of (2) is independent of $y$ by translation, and is convergent in $R^{n}$ if $N>2 n$. Thus the left hand side of (2) is bounded by a constant times $\left(1+|\tau \delta|^{N / 2}\right)^{-1}$, which is in turn bounded by a constant times $(|y|+1)^{-\alpha-\beta}$, after we substitute the value of $\delta$ which depends on $y$.

Next, we consider the remaining part, over $B(y)$, of the integral in (1). Then

$$
\begin{aligned}
& \int_{B(y)}(|x|+1)^{-\alpha}\left(1+|\tau(x-y)|^{N}\right)^{-1} d x \\
& \quad \leqq \int_{B(y)}(|x|+1)^{-\alpha} d x \leqq(\max \{1,|y|-\delta\})^{-\alpha} \text { meas } B(y) .
\end{aligned}
$$

From the $\beta$-condition on $E$,

$$
\text { meas }(E \cap\{x: a \leqq|x|<a+1\}) \leqq C(1+a)^{-\beta},
$$

we have

$$
\text { meas } B(y) \leqq \operatorname{const}(|y|+1)^{-r}\left[(|y|+1)^{-(\beta-\gamma)}(|y|+1)^{2(\alpha+\beta) / N}\right] \text {, }
$$

which is less than a constant times $(|y|+1)^{-r}$ if $N>2(\alpha+\beta)(\beta-\gamma)^{-1}$. 
If we now insert this estimate for meas $B(y)$ into the left hand side of (3), then (3) is bounded by a constant times $(|y|+1)^{-\alpha-r}$. The combined estimates for (2) and (3) complete the proof of inequality (1).

Lemma 2.2. If $\gamma\langle\beta, \tau\rangle \tau_{0}$, and $N$ sufficiently is large, then there is an $\varepsilon_{0}$ and $a K$ such that

$$
\int_{E}|x-y|^{-8}\left(1+|\tau(x-y)|^{N}\right)^{-1} d y \leqq K(|y|+1)^{-r}
$$

where $0<\varepsilon<\varepsilon_{0}, \varepsilon_{0}=\varepsilon_{0}(\beta, \gamma)$, and $K=K\left(\beta, \gamma, \tau_{0}, N\right)$.

Proof. Set $B(y)=B(y, 1) \cap E$, where $B(y, 1)$ is the ball center at $y$ and radius equal to 1 .

The integral on the left hand side of (4), integrated only over the set $B(y)$, is bounded by

$$
\int_{B(y)}|x-y|^{-\varepsilon} d y \leqq(\operatorname{meas} B(y))^{1-2^{-m}}\left\{\int_{B(y)}|x-y|^{-^{m_{\varepsilon}}} d y\right\}^{2^{-m}},
$$

if we apply the Schwarz inequality $m$ times to the left side of (5). From the $\beta$-condition on $E$, we have

$$
\text { meas } B(y) \leqq \text { const }(|y|+1)^{-\beta} \text {. }
$$

Now pick $m$ so large that $\beta\left(1-2^{-m}\right)>\gamma$, then pick $\varepsilon_{0}$ so small that $2^{m} \varepsilon_{0}<m$. The choice of $\varepsilon_{0}$ makes the integral on the right hand side of (5) convergent for $\varepsilon<\varepsilon_{0}$, and leads to the estimate of a constant times $(|y|+1)^{-r}$ for (5).

To estimate the integral in (4) over $B^{\prime}(y)=E-B(y)$, we use Lemma 2.1 with $\alpha=0$ to get the estimate of a constant times $(|y|+1)^{-r}$. This completes the proof of inequality (4).

Let $G^{(i)}(\lambda, z, x)$ be the iterates of the Green's function on $E$, which was constructed in Theorem 1.7, these iterates are defined by

$$
G^{(i)}(\lambda, z, x)=G\left(G^{(i-1)}(\lambda, z, x)\right) ; G^{(1)}(\lambda, z, x)=G(\lambda, z, x) .
$$

Lemma 2.3. If $\gamma\langle\beta, \tau\rangle \tau_{0}$, and $N$ is sufficiently large, then the iterate $G^{(2)}(\lambda, y, x)$ satisfies

(7) $\left|G^{(2)}\left(-\tau^{2}, y, x\right)\right| \leqq M\left(1+|\tau(x-y)|^{N}\right)^{-1}(|x|+1)^{-\gamma / 2}(|y|+1)^{-\gamma / 2}$ for $\operatorname{dim} E=2$, where $M$ is independent of $x$ and $y$.

Proof. From Lemma 1.9 and the triangle inequality, we have 
( 8 )

$$
\begin{aligned}
& \left|G^{(2)}\left(-\tau^{2}, y, x\right)\right| \leqq M\left(1+|\tau(x-y)|^{N / 2}\right)^{-1} \\
& \quad \times \int_{E}|\tau(x-z)|^{2-n-\varepsilon}|\tau(z-y)|^{2-n-\varepsilon}\left(1+|\tau(x-z)|^{N / 2}\right)^{-1} \\
& \quad \times\left(1+|\tau(z-x)|^{N / 2}\right)^{-1} d z .
\end{aligned}
$$

If we apply the Schwarz inequality, then the integral in (8) can be estimated by

$$
\left\{\int_{E}|\tau(x-z)|^{4-2 n-2 \varepsilon}\left(1+|\tau(x-z)|^{N}\right)^{-1} d z\right\}^{1 / 2}
$$

times exactly the same integral with $x$ replaced by $y$. If in expression (9), $N>n, 2 n+2 \varepsilon-4<n$ (i.e., $n=2$ or $n=3$ ), and $E$ is replaced by $R^{n}$, then the expression is seen to be bounded by a constant, which is independent of $x$. This completes the proof of inequality (6). The estimate for expression (9) can be improved in the case $n=2$. Lemma 2.2 implies, if $\varepsilon$ is sufficiently small, that (9) is bounded by const $(|x|+1)^{-r / 2}$. Combining all estimates, we have inequality (7) when $\operatorname{dim} E=2$.

Theorem 2.4. If $\gamma\langle\beta, \tau\rangle \tau_{0}$, and $N$ sufficiently large, then

$$
\iint_{E} G^{(2 k)}\left(-\tau^{2}, x, y\right)^{2} d x d y \leqq M \sum_{i=1}^{\infty} i^{-4 k \gamma}, \operatorname{dim} E=2,
$$

and

$$
\iint_{E} G^{(2 k)}\left(-\tau^{2}, x, y\right)^{2} d x d y \leqq M \sum_{i=1}^{\infty} i^{-2 k \gamma}, \operatorname{dim} E=3,
$$

where $k$ is a positive integer, and $M$ is a constant.

Proof. We will only consider the case $\operatorname{dim} E=3$ - the other case is similar.

We can show using induction, the triangle inequality, Lemma 2.3, and Lemma 2.1 that

$$
G^{(2 k)}\left(-\tau^{2}, x, y\right) \leqq \text { const }(|x|+1)^{-(k-1) r}\left\{\left(1+|\tau(x-y)|^{N}\right)^{2^{1-k}}\right\}^{-1}
$$

for $k$ a positive integer ( $k=1$ is Lemma 2.3).

If we square both sides of the above inequality, integrate over $E$, and apply Lemma 2.1 , then

$$
\int_{E} G^{(2 k)}\left(-\tau^{2}, x, y\right)^{2} d x \leqq \text { const }(|y|+1)^{-(2 k-1) \gamma} .
$$

Integrating again over $E$, we have

$$
\int_{E} \int_{E} G^{(2 k)}\left(-\tau^{2}, x, y\right)^{2} d x d y \leqq \text { const } \sum_{i=0}^{\infty} \int_{E_{i}}(|y|+1)^{-(2 k-1) r} d y
$$


where $E_{i}=\{x \in E: i \leqq|x|<i+1\}$. This expression is in turn

$$
\leqq \text { const } \sum_{i=0}^{\infty}(i+1)^{-(2 k-1) r} \text { meas }\left(E_{i}\right) \leqq M \sum_{i=0}^{\infty}(i+1)^{-2 k \gamma},
$$

since meas $\left(E_{i}\right) \leqq$ const $(i+1)^{-\beta}$ by the $\beta$-condition. This completes the theorem.

COROLLARY 2.5. The iterate $G^{(2 k)}\left(-\tau^{2}, x, y\right)$ is a Hilbert-Schmidt kernel, in $L_{2}(E)$ for $\tau>\tau_{0}$, provided $4 k>\beta^{-1}$ in case $\operatorname{dim} E=2$, and $2 k>\beta^{-1}$ in case $\operatorname{dim} E=3$, where $\beta$ is given by the $\beta$-condition.

Proof. Pick $\gamma<\beta$ but sufficiently close, and apply Theorem 2.4.

\section{The eigenvalues of the problem.}

THEOREM 3.1. Let $\lambda_{j}$ be the set of eigenvalues, given in Theorem 1.6, for $T$ on the set $E$. Then the series

$$
\sum_{j=1}^{\infty} \lambda_{j}^{-4 k}
$$

converges for $\operatorname{dim} E=2$ if $4 k>\beta^{-1}$, and for $\operatorname{dim} E=3$ if $2 k>\beta^{-1}$.

Proof. Let $u_{j}$ be the orthonormal set of eigenfunctions, given by Theorem 1.6, corresponding to the eigenvalues $\lambda_{j}$. If $\lambda<0$ is not in the spectrum of $T$, then it follows from $T u_{j}=\lambda_{j} u_{j}$ that $G u_{j}=$ $\left(\lambda_{j}+\tau^{2}\right)^{-1} u_{j}$ where $\lambda=-\tau^{2}$. Thus for the $2 k$ iterate of $G$ we have $G^{(2 k)} u_{j}=\left(\lambda_{j}+\tau^{2}\right)^{-2 k} u_{j}$. However, from Corollary 2.5 we have that the kernel $G^{(2 k)}\left(-\tau^{2}, x, y\right)$ of $G^{(2 k)}$ is Hilbert-Schmidt for $\operatorname{dim} E=2$ if $4 k>\beta^{-1}$, and for $\operatorname{dim} E=3$ if $2 k>\beta^{-1}$. It follows from Agmon [2, Theorem 12.18] that the Hilbert-Schmidt double norm

$$
\sum_{j=1}^{\infty}\left(\lambda_{j}+\tau^{2}\right)^{-4 k}<\infty .
$$

But since all the eigenvalues of $T$ are positive, we have our desired result.

COROLlaRY 3.2. If $k$ satisfies the conditions of Theorem 3.1, then the function $N(\lambda)=\sum_{\lambda_{j} \leqq \lambda} 1$ satisfies

$$
\lambda^{-4 k} N(\lambda) \leqq \text { constant } .
$$

Proof. Since the sequence $\left\{\lambda_{j}^{-4 k}\right\}$ is nonincreasing and $\sum \lambda_{j}^{-4 k}<$ $\infty$, by Theorem 3.1, we have $\lambda_{j}^{-4 k}=O\left(j^{-1}\right)$. Hence $\lambda_{j} \geqq M j^{1 / 4 k}$ for some $M>0$, and therefore 


$$
N(\lambda)=\sum_{\lambda \leq \lambda} 1 \leqq \sum_{j \leqq\left(M^{-1}\right)^{4 k}} 1=\left(M^{-1} \lambda\right)^{4 k} ;
$$

this shows that $\lambda^{-4 k} N(\lambda) \leqq$ const, as asserted.

\section{REFERENCES}

1. R. A. Adams, The Rellich-Kondrachov theorem for unbounded domains, Archive Rat. Mech. Anal., 29 (1968), 390-394.

2. S. Agmon, Lectures on Elliptic Boundary Value Problems, Van Nostrand, Princeton, New Jersey, 1965.

3. F. H. Brownell, Spectrum of the static potential Schrödinger equations over $E_{m}$, Ann. of Math., 54 (1954), 554-594.

4. C. Clark, An embedding theorem for function spaces, Pacific J. Math., 19 (1966), 243-251.

5. - An asymptotic formula for the eigenvalues of the Laplacian operator in an unbounded domain, Bull. Amer. Math. Soc., 72 (1966), 709-712.

6. C. Clark and D. E. Hewgill, One can hear whether a drum has finite area, Proc. Amer. Math. Soc., 18 (1967), 236-237.

7. L. Gårding, On the asymptotic distribution of the eigenvalues and eigenfunctions of elliptic partial differential operators, Math. Scand., 1 (1953), 237-255.

8. D. Hewgill, On the eigenvalues of the Laplacian in an unbounded domain, Arch. Rat. Mech. Anal., 27 (1967), 153-164.

9. M. Krzyżański, Partial Differential Equations on Second Order, Vol. I, PWNPolish Scientific Publishers, Warszawa, 1971.

10. K. Maurin, Methods of Hilbert Spaces, PWN-Polish Scientific Publishers, Warszawa, 1967.

11. G. V. Rozenbljum, On the distribution of eigenvalues of the first boundary value problem in unbounded domains, Soviet Math. Dokl., 12 (1971), 1539-1542.

Received February 14, 1973 and in revised form June 25, 1973. This research was sponsored by the National Research Council of Canada under grant N.R.C. A7558.

THE UNIVERSITY OF VICTORIA 


\section{PACIFIC JOURNAL OF MATHEMATICS}

\section{EDITORS}

RICHARD ARENS (Managing Editor)

University of California

Los Angeles, California 90024
J. DUGUNDJI*

Department of Mathematics

University of Southern California

Los Angeles, California 90007

D. Gilbarg and J. Milgram

Stanford University

Stanford, California 94305
University of Washington

Seattle, Washington 98105

ASSOCIATE EDITORS
E. F. BECKENBACH
B. H. NeumanN
F. WoLF
K. YosHIDA

\section{SUPPORTING INSTITUTIONS}

\author{
UNIVERSITY OF BRITISH COLUMBIA \\ CALIFORNIA INSTITUTE OF TECHNOLOGY \\ UNIVERSITY OF CALIFORNIA \\ MONTANA STATE UNIVERSITY \\ UNIVERSITY OF NEVADA \\ NEW MEXICO STATE UNIVERSITY \\ OREGON STATE UNIVERSITY \\ UNIVERSITY OF OREGON \\ OSAKA UNIVERSITY
}

\author{
UNIVERSITY OF SOUTHERN CALIFORNIA \\ STANFORD UNIVERSITY \\ UNIVERSITY OF TOKYO \\ UNIVERSITY OF UTAH \\ WASHINGTON STATE UNIVERSITY \\ UNIVERSITY OF WASHINGTON
* * * *
AMERICAN MATHEMATICAL SOCIETY \\ NAVAL WEAPONS CENTER
}

The Supporting Institutions listed above contribute to the cost of publication of this Journal, but they are not owners or publishers and have no responsibility for its content or policies.

Mathematical papers intended for publication in the Pacific Journal of Mathematics should be in typed form or offset-reproduced, (not dittoed), double spaced with large margins. Underline Greek letters in red, German in green, and script in blue. The first paragraph or two must be capable of being used separately as a synopsis of the entire paper. Items of the bibliography should not be cited there unless absolutely necessary, in which case they must be identified by author and Journal, rather than by item number. Manuscripts, in duplicate if possible, may be sent to any one of the four editors. Please classify according to the scheme of Math. Rev. Index to Vol. 39. All other communications to the editors should be addressed to the managing editor, or Elaine Barth, University of California, Los Angeles, California, 90024.

100 reprints are provided free for each article, only if page charges have been substantially paid. Additional copies may be obtained at cost in multiples of 50 .

The Pacific of Journal Mathematics is issued monthly as of January 1966. Regular subscription rate: $\$ 72.00$ a year (6 Vols., 12 issues). Special rate: $\$ 36.00$ a year to individual members of supporting institutions.

Subscriptions, orders for back numbers, and changes of address should be sent to Pacific Journal of Mathematics, 103 Highland Boulevard, Berkeley, California, 94708.

PUBLISHED BY PACIFIC JOURNAL OF MATHEMATICS, A NON-PROFIT CORPORATION

Printed at Kokusai Bunken Insatsusha (International Academic Printing Co., Ltd.), 270, 3-chome Totsuka-cho, Shinjuku-ku, Tokyo 160, Japan.

* C. R. DePrima California Institute of Technology, Pasadena, CA 91109, will replace J. Dugundji until August 1974.

Copyright (C) 1973 by Pacific Journal of Mathematics

Manufactured and first issued in Japan 


\section{Pacific Journal of Mathematics}

\section{Vol. 51, No. $2 \quad$ December, 1974}

Robert F. V. Anderson, Laplace transform methods in multivariate spectral theory .................................................. 339

William George Bade, Two properties of the Sorgenfrey plane . . . . . . . . . . . . 349

John Robert Baxter and Rafael Van Severen Chacon, Functionals on continuous

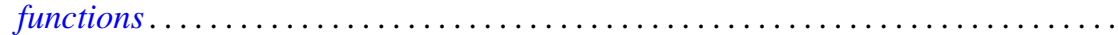

Phillip Wayne Bean, Helly and Radon-type theorems in interval convexity

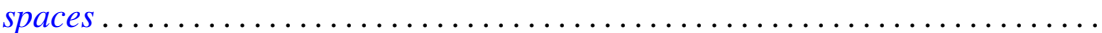

James Robert Boone, On k-quotient mappings $\ldots \ldots \ldots \ldots \ldots \ldots \ldots \ldots \ldots$

Ronald P. Brown, Extended prime spots and quadratic forms . . . . . . . . . . . .

William Hugh Cornish, Crawley's completion of a conditionally upper continuous lattice .............................................

Robert S. Cunningham, On finite left localizations ...................

Robert Jay Daverman, Approximating polyhedra in codimension one spheres

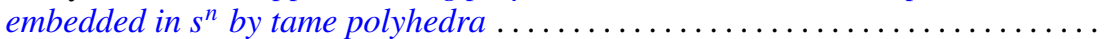

Burton I. Fein, Minimal splitting fields for group representations . . . . . . . . . . . .

Peter Fletcher and Robert Allen McCoy, Conditions under which a connected

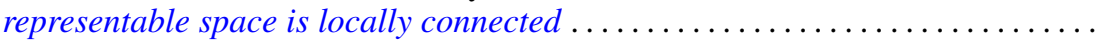

Jonathan Samuel Golan, Topologies on the torsion-theoretic spectrum of a noncommutative ring...

Manfred Gordon and Edward Martin Wilkinson, Determinants of Petrie matrices.

Alfred Peter Hallstrom, A counterexample to a conjecture on an integral condition for determining peak points (counterexample concerning peak points)........

E. R. Heal and Michael Windham, Finitely generated $F$-algebras with applications to Stein manifolds.

Denton Elwood Hewgill, On the eigenvalues of a second order elliptic operator in an unbounded domain ............................

Charles Royal Johnson, The Hadamard product of $A$ and $A^{*}$.

Darrell Conley Kent and Gary Douglas Richardson, Regular completions of Cauchy spaces.

Alan Greenwell Law and Ann L. McKerracher, Sharpened polynomial approximation

Bruce Stephen Lund, Subalgebras of finite codimension in the algebra of analytic functions on a Riemann surface. .

Robert Wilmer Miller, TTF classes and quasi-generators . .

Roberta Mura and Akbar H. Rhemtulla, Solvable groups in which every maximal partial order is isolated ....

Isaac Namioka, Separate continuity and joint continuity...

Alan Saleski, Entropy of self-homeomorphisms of statistical pseudo-metric

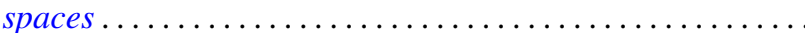

H. A. Seid, Cyclic multiplication operators on $L_{p}$-spaces .....

H. B. Skerry, On matrix maps of entire sequences ............

John Brendan Sullivan, A proof of the finite generation of invariants of a normal

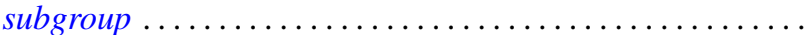

John Griggs Thompson, Nonsolvable finite groups all of whose local subgroups are

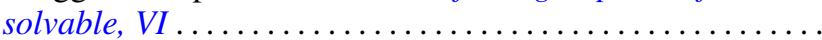

